\title{
Single nucleotide polymorphisms and unacceptable late toxicity in breast cancer adjuvant radiotherapy: a case report
}

This article was published in the following Dove Press journal:

Breast Cancer - Targets and Therapy

29 May 2017

Number of times this article has been viewed

\author{
Grazia Lazzari' \\ Maria lole Natalicchio ${ }^{2}$ \\ Angela Terlizzi ${ }^{3}$ \\ Francesco Perri ${ }^{4}$ \\ Giovanni Silvano' \\ 'Radiation Oncology Unit, San \\ Giuseppe Moscati Hospital, Taranto, \\ ${ }^{2}$ Molecular Biology Laboratory, \\ Pathological Anatomy Department, \\ Ospedali Riuniti, Foggia, ${ }^{3}$ Medical \\ Physic Unit, San Giuseppe Moscati \\ Hospital, ${ }^{4}$ Medical Oncology Unit, \\ Presidio Ospedaliero Centrale - \\ Santissima Annunziata, Taranto, Italy
}

Correspondence: Grazia Lazzari Radiation Oncology Unit, San Giuseppe Moscati Hospital, Str Per Martina Franca, 74100 Taranto, Italy

Tel +39994585721

Email lazzarigrazia@gmail.com
Background: There has recently been a strong interest in the inter-individual variation in normal tissue and tumor response to radiotherapy (RT), because tissue radiosensitivity seems to be under genetic control. Evidence is accumulating on the role of polymorphic genetic variants, such as single nucleotide polymorphisms (SNPs) that could influence normal tissue response after radiation. The most studied SNPs include those in genes involved in DNA repair (single- and double-strand breaks, and base excision) and those active in the response to oxidative stress.

Case report: We present the case report of a 60 -year-old woman with early breast cancer who underwent adjuvant hormone therapy and conventional radiotherapy, and subsequently developed unacceptable cosmetic toxicities of the irradiated breast requiring a genetic test of genes involved in DNA repair mechanisms. The patient was found to be heterozygous for G28152A (T/C) and C18067T (A/G) mutations in X-ray repair cross-complementing group 1 $(X R C C 1)$ and $3(X R C C 3)$, respectively, homozygous for $\mathrm{A} 313 \mathrm{G}(\mathrm{G} / \mathrm{G})$ mutation in glutathione $\mathrm{S}$ transferase Pi 1 (GSTP1), and wild-type for A4541G (A/A) in XRCC3 and G135C (G/G) in RAD51 recombinase.

Conclusion: The role of SNPs should be taken into account when a severe phenomenon appears in normal tissues after radiation treatment, because understanding the molecular basis of individual radiosensitivity may be useful for identifying moderately or extremely radiosensitive patients who may need tailored therapeutic strategies.

Keywords: radiosensitivity, SNPs, fibrosis, DNA repair

\section{Introduction}

Adjuvant radiotherapy (RT) in breast cancer after conservative surgery has become a widely accepted standard of care for patients with early breast cancer. Early Breast Cancer Trialists' Collaborative Group meta-analysis has clearly demonstrated that adjuvant RT after breast-conserving surgery (BCS) reduces the 10-year risk of any locoregional or distant first recurrence from $35 \%$ to $19.3 \%(2 p<0.00001)$ and the 15 -year risk of breast cancer deaths from $25.2 \%$ to $21.4 \%(2 p=0.00005) .{ }^{1}$ However, RT acts on tumors and the surrounding normal tissues but could cause acute and late side effects, which could range from mild to severe depending on fractionation size and $\alpha / \beta$ ratio of involved tissues. ${ }^{2}$ Acute normal tissue toxicity that appears as erythema, edema, swelling, and epithelial stripping is generally a transient phenomenon, while late normal tissue toxicities, such as telangiectasias, tissue fibrosis with functional tissue impairment, and skin retraction with poor cosmesis, are progressive and permanent findings. These acute and late normal tissue radiation-induced toxicities are strongly related to individual radiosensitivity that could vary widely from patient to patient. 
Tissue hypoxia, drugs employed, and genetic features may also affect the clinical tissue radiosensitivity after a normal fractionated schedule. Drugs such as chemotherapy or hormone therapy have been demonstrated to enhance subcutaneous fibrosis after radiotherapy as reported by Skoczylas et al and Bentzen et al who analyzed the impact of adjuvant cyclophosphamide, methotrexate, and fluorouracil (CMF) or tamoxifen on cosmesis. ${ }^{3,4}$ On the other hand, the use of aromatase inhibitors seems to exert a protective effect on post-radiation-induced lung fibrosis. ${ }^{5}$ Once pharmacologic causes have been excluded, radiation-induced late tissue injury could be due to altered DNA sequences such as single nucleotide polymorphisms (SNPs) affecting genes such as $\mathrm{X}$-ray repair cross-complementing group 1 and 3 (XRCC1 and $X R C C 3)$, glutathione $\mathrm{S}$ transferase Pi 1 (GSTP1), and $R A D 51$ recombinase.

We report a case of a breast cancer patient with SNPs in the aforementioned genes who developed an unpredictable severe or grade 3 (scored with SOMA-LENT system) ${ }^{6}$ subcutaneous fibrosis and skin telangiectasias with atrophy that evolved into a poor cosmetic outcome after adjuvant RT to the breast.

\section{Case report}

In 2012, a 60-year-old woman came to our attention with left breast cancer. In her clinical history, there were no autoimmune and vascular diseases, or diabetes. She had a moderately differentiated (grade 2) ductal carcinoma, with high expression of estrogen and progesterone receptors. She was treated with BCS on her external superior mammary quadrant and sentinel lymph node biopsy (stage pT1c pN0sn Er+ PR+ Her 2+), followed by radiotherapy and adjuvant letrozole. In February 2012, she received adjuvant radiotherapy to the whole residual breast with three-dimensional (3D) conformal technique, $6 \mathrm{MV}$ photons, and multileaf collimator customized beams with a standard fractionation of 2 Gy ( 50 Gy total), followed by a 10 Gy boost with a 12 $\mathrm{MeV}$ electron beam to the tumor bed.

During the treatment, she did not develop any acute skin reaction. Ten months later, the patient returned to our attention when complaining of hemorrhagic skin of the central-inferior quadrant of the irradiated breast, which was surrounded by multiple and frail telangiectasias. During the first 3 weeks, the lesion began to enlarge, and ultimately consisted of a big hemorrhagic morula, surrounded by several pustules, and outlined by an edematous circular edge (Figure 1). Two weeks later, the burst pustules released hemosiderinic products onto the skin. Two months later, the initially involved area became pale and avascular, and was covered by a transparent epithelial coat surrounded by multiple frail and thin telangiectasias (Figure 2). These phenomena recurred every 4 to 6 months, involving at least the entire irradiated breast. The breast was severely retracted and distorted with edema of the subcutaneous plane and induration in the central part near the nipple area, with peripheral telangiectasias (Figure 3). We decided to review the RT treatment plan for possible errors in the radiation planning and delivery, and to check for possible under-estimated high hot spot (highest skin dose) areas, which could explain this phenomenon. However, the execution of the RT treatment was correct; in the evaluation plan the configuration of the isodoses, the dose/fraction, and the effective total dose administered were accurate. In the revision of the plan, only $105 \%$ little hot spot areas in the upper external part of the breast were recorded, while the area of the boost was in the superior external quadrant, outside the involved fibrotic breast.

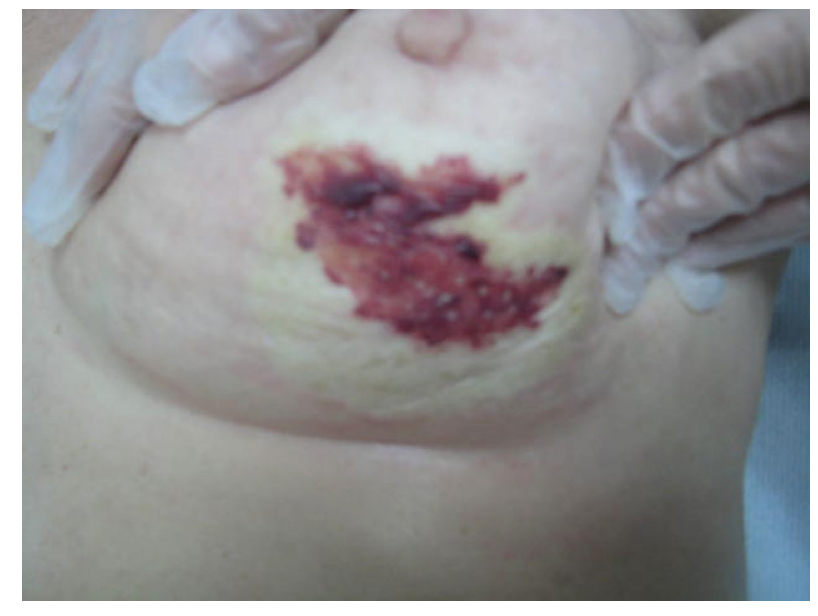

Figure I The picture shows the lesion characterized by a big morula and many pustules with a frail roof within hemorrhagic fluid, outlined by an edematous circular edge.

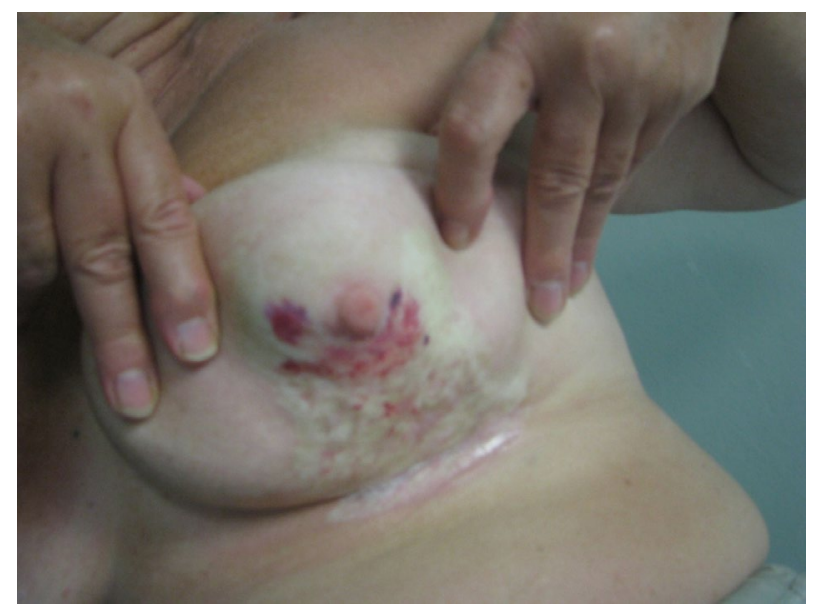

Figure 2 Two months after initial presentation, the involved area became pale, avascular, and covered by a transparent epithelial coat surrounded by multiple frail and thin telangiectasias with retraction of the skin in the inferior quadrants of the breast. 


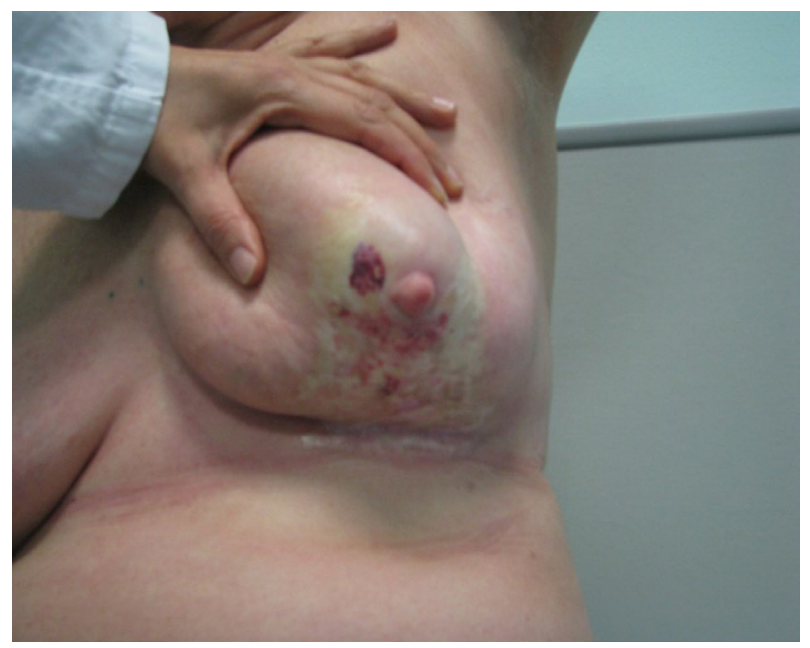

Figure 3 The picture shows a severely retracted and distorted breast with edema of the subcutaneous tissues as well as induration in the central part over the nipple area with peripheral telangiectasias.

To exclude a local relapse and to avoid a pressed trauma to the affected breast by the mammography, a breast magnetic resonance imaging (MRI) was prescribed. Breast MRI showed no relapse and a contrast enhancement of the dermal-epidermal surface with thickening of the skin contour as shown in Figure 4A and B. To exclude the suspicion of an active autoimmune connective-tissue skin syndrome, the patient underwent patch biopsy of the involved area. The biopsy revealed findings of chronic inflammatory diseases, such as panniculitis and chronic radiodermatitis, characterized by fibrosis and dermo-epidermal strippings, telangiectasias with thin and frail vessels (Figure 5). At this point, we hypothesized the possible role of polymorphisms in genes involved in DNA repair mechanisms. We therefore tested for SNPs in genes of interest on a peripheral blood sample using polymerase chain reaction and pyrosequencing. The tested genes were GSTP1 (A313G), XRCC1 (G28152A), XRCC3 (A4541G and C18067T), RAD51 (G135C), CYP3A4*1B, and CYP19A1 (C268T and G410T). The result showed heterozygous mutations in XRCC1 G28152A (T/C) and XRCC3 C18067T (A/G), homozygous mutation in GSTP1 A313G $(\mathrm{G} / \mathrm{G})$, and wild-type $X R C C 3$ A4541G (A/A) and RAD51 G135C (G/G).
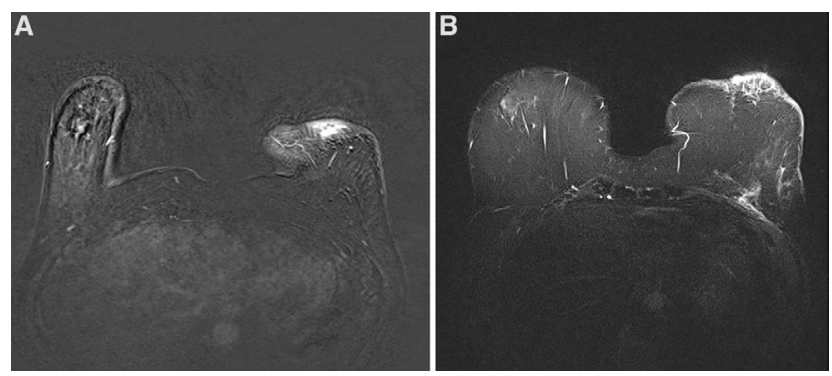

Figure 4 Magnetic resonance images of the breast show a contrast enhancement of the dermal-epidermal surface with thickening of the skin contour. (A) Is in the caudal part where there is oedema, $(\mathbf{B})$ is in the middle part of the lesion where there are more vessels.

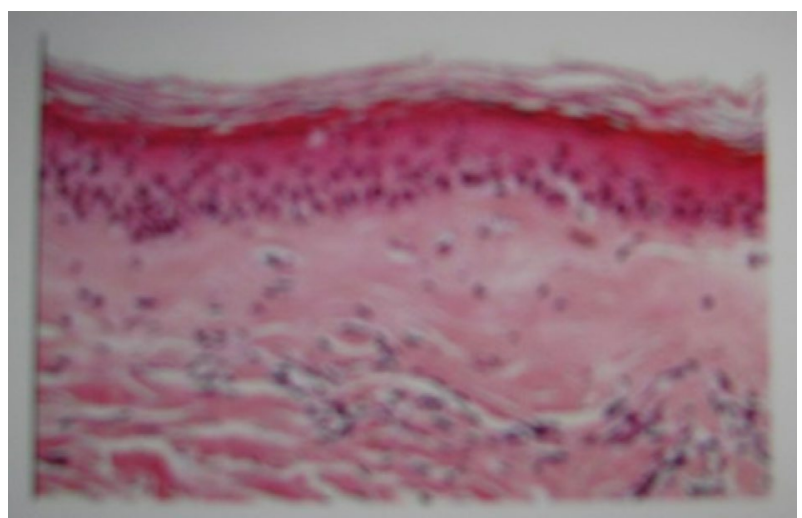

Figure 5 Patch biopsy of the involved area with findings of chronic inflammatory disease evidenced as panniculitis and chronic radiodermatitis.

The patient was informed that she was a carrier of these genetic alterations, and that they could be responsible for the late toxicity and worse cosmetic outcome after radiotherapy. She was observed with close follow-up and delivery of the necessary topical medications with the aim of curing the radiation-induced skin damage. Written informed consent was obtained from the patient for publication of the case details and images in this paper.

\section{Discussion}

BCS followed by adjuvant RT is replacing mastectomy as the standard treatment for early breast cancer. Large clinical trials with a median follow-up of 20-30 years have shown that BCS plus RT is safe and effective, achieving an excellent cosmetic outcome in approximately $80 \%$ of patients. ${ }^{7}$ Standard adjuvant RT consists of whole breast radiotherapy with 50 Gy in 25 daily fractions with or without a boost of 10 Gy. Nowadays, it is performed worldwide with conformal $3 \mathrm{D}$ forward or inverse-planned techniques. Nevertheless, the benefit of RT is also associated with adverse effects in terms of acute and late normal tissue toxicity as summarized in the "therapeutic ratio" concept. ${ }^{2}$ Radiation-induced adverse reactions are commonly classified as early or late effects, depending on the time of clinical symptom manifestation and the $\alpha / \beta$ ratio of the involved tissue. Acute-early effects occur within a few weeks after starting RT. They often involve rapidly proliferating tissues such as normal epithelial tissues, which are characterized by high $\alpha / \beta$ ratio. These effects are usually transient and related to an interruption of epithelial-cell generation, followed by thinning or denudation of epithelia and consequent healing. Chronic-late effects develop in months to some years after completion of RT, as in our patient. They involve tissues with a low $\alpha / \beta$ ratio and slow cell turnover. ${ }^{8,9}$ These events are usually more persistent and troublesome, 
including chronic inflammatory processes, vascular changes, fibrosis, and atrophy. Radiobiology tells us that tissue radiosensitivity depends on the $\alpha / \beta$ ratio of the tissue and on the 5 Rs (repair, reassortment, repopulation, reoxygenation, and radiosensitivity) of the irradiated tissues, but it may also be influenced by environmental factors such as drugs. ${ }^{10}$ In breast cancer, adjuvant chemotherapy and hormone therapy have been acknowledged to influence radiosensitivity of the residual breast or the underlying irradiated normal tissues. Bentzen et al reported that the use of adjuvant CMF was responsible for the development of subcutaneous fibrosis after RT, showing a "shift on the left" of the plotted normal-tissue complication dose-response curve. ${ }^{11}$ Regarding the role of hormone therapy, the question remains controversial. The use of tamoxifen seems to cause lung fibrosis after adjuvant breast radiotherapy, while aromatase inhibitors seem to protect from lung fibrosis after adjuvant breast radiotherapy, ${ }^{4,5}$ However, our patient did not receive chemotherapy or tamoxifen treatment. Thus, in the absence of these "external factors", as in our patient, a possible genetic explanation of an altered normal tissue radiosensitivity due to the presence of these SNPs should be hypothesized. Thus, we have analyzed all the available radiogenomic studies concerning the impact of SNPs in DNA repair genes in radiation-induced damage. Ionizing radiation has been demonstrated to produce its biological effects by generation of short-lived but highly reactive DNA radicals that evolve into stable and long-lived DNA lesions such as single-strand breaks and double-strand breaks (DSBs), leading to cell death. ${ }^{12,13}$ Damage repair is mediated by hundreds of genes involved in DNA repair machinery, such as DNA damage recognition, DNA repair pathways (including nonhomologous end joining of DSBs, homologous recombination repair of DSBs, and base excision repair of other types of single and simple clustered lesions), activation of cell-cycle checkpoints, signal transduction, and oxidative stress responses, as well as the apoptotic and premature senescence pathways. ${ }^{14}$ Allelic variations in the sequence of these genes, such as brief repeating sequences or SNPs, have been considered by radiogenomic studies to play a role in the development of post-radiation acute and late toxicities. ${ }^{15}$ The most studied SNPs are found in a few genes, including GSTP1 (A313G), XRCC1 (G28152A, rs25487), XRCC3 (A4541G), $X R C C 3$ (C18067T), and RAD51 (G135C).

Our patient showed a mutation of $X R C C 1$ (T/C G28152A rs25487) in the heterozygous state. $X R C C 1$ encodes a protein involved in single base excision-repair (BER), which is the most common repair after damage induced by X-rays, reactive oxygen species, and alkylating agents. $\mathrm{XRCC} 1$ is a scaffold protein that coordinates both with the initial and late steps of abasic site restoration via multiple protein-protein interactions. ${ }^{16}$ By accurate analyses of radiogenomic studies from the literature, this SNP seems to play an important role in increasing the sensitivity to ionizing radiations. ${ }^{17,18}$ Moullan et al have hypothesized that distinct combinations of XRCC1 polymorphisms could be associated with the possibility of developing an adverse response to RT. ${ }^{19}$ A recent meta-analysis of several studies investigating the role of XRCC1 polymorphisms, in mostly Caucasian patients, on acute and late normal tissue reactions to irradiation revealed controversial findings. ${ }^{20} \mathrm{On}$ the other hand, studies have found that SNPs that are associated in BER genes such as APE1 may have a protective effect against radiation-induced acute and late damage in Caucasian breast cancer patients. ${ }^{21,22}$ Thus, the predictive value of $X R C C 1$ polymorphisms on late postradiation breast toxicity remains unclear, in spite of many single investigational attempts made to validate this correlation. In fact, Andreassen et al, in their analysis, concluded that XRCC1 Arg399Arg genotype could be associated with post-radiation breast fibrosis, but in a follow-up cohort study they were unable to confirm these data. ${ }^{23,24}$

Most recently, a study investigating the predictive value of ten SNPs in four genes involved in DNA damage repair, including XRCC1 rs25487, on late toxicity after radiotherapy in breast cancer patients has been conducted using a state of the art design with replications. In the discovery cohorts, these SNPs were not associated with either skin toxicity or fibrosis $(p<0.05)$, and in the replication cohorts, none of them were statistically significantly associated with any of the late toxicity endpoints investigated. ${ }^{25}$ However, in vitro and in vivo studies have found a strong link between haplotypes of $X R C C 1$ and late radiation injury in irradiated breast. In a prospective study, XRCC1 SNPs (rs1799782 and rs25487) were significantly associated with adverse reactions to RT, such as altered pigmentation $(p=0.03)$ and telangiectasia $(p=0.01) .{ }^{26}$ Moreover, it is still unclear if late post-radiation sequelae could be under multiple-gene or single-gene control. Azria et al, in an "in vitro" study, demonstrated that four or more SNPs in DNA repair genes controlled the development of severe late radiation-induced side effects, while Giotopoulos et al observed that fibrosis and telangiectasia could be under separate genetic control. ${ }^{27,28}$ The authors showed that the XRCC1 Arg399GIn SNP was associated specifically with late telangiectasia but not fibrosis, which was associated with transforming growth factor B1 509T (TGF-B1 509T). ${ }^{28}$

The genetic signature of our patient included a heterozygous XRCC3 (A/G) C18067T mutation associated with 
a wild-type $X R C C 3$ A4541G. $X R C C 3$ is also a member of the RAD51-related protein family that participates in homologous recombination repair of DSBs and plays an important role in maintaining chromosome stability. Noteworthy, DNA DSBs occur frequently after irradiation and are considered potent inducers of mutations and cell death. ${ }^{29,30}$ The XRCC3 C18067T mutation found in our patient is also known as $X R C C 3$ p.Thr241Met (rs861539) and is the most commonly reported polymorphism in XRCC3. The XRCC3 protein has a low repair activity and a high in vitro radiosensitivity that promotes chromosome instability and cell's inability to repair radiation injury. ${ }^{31}$ Several meta-analyses have also demonstrated an association between the XRCC3 p.Thr241Met polymorphism and the risk of breast cancer development, mainly in Asian population. ${ }^{32}$ The association between $X R C C 3$ polymorphisms and the risk of radiationinduced adverse effects has been evaluated in many studies using scoring systems or Q-Q plots to predict normal tissue response in radiosensitive patients, but the results have been controversial. ${ }^{26}$

A robust support for our findings comes from a recent meta-analysis that found the $X R C C 3 \mathrm{p}$. Thr241Met polymorphism to be significantly associated with early adverse effects, such as acute skin toxicity and mucositis, mostly in patients irradiated for head and neck cancer. ${ }^{33}$ This SNP was significantly correlated with an increased risk of adverse effects in head and neck $(p=0.0003)$ and breast $(p=0.04)$ irradiation. In a subgroup analysis, the authors observed a significant association between this specific SNP and radiotherapyinduced fibrosis $(p=0.05)$. Moreover, the co-expression of the aforementioned SNP and the XRCC3 rs861534 $\left(r^{2}=\right.$ $0.96)$ seems to increase the risk of subcutaneous fibrosis. ${ }^{23}$

Our patient also harbored homozygous GSTP1 A313G $(\mathrm{G} / \mathrm{G})$ mutation. The glutathione S-transferases are a family of enzymes that play important roles in detoxification by catalyzing the conjugation of many hydrophobic and electrophilic compounds with reduced glutathione and thus play an important role in cell resistance to oxidative stress. ${ }^{34}$ Falvo et al showed that moderate fibrosis (>grade 2 ) or fatty necrosis were more frequent in patients with than without GSTP1 mutation (64\% vs. $38 \% ; p=0.047)$, concluding that this mutation could be useful for predicting late toxicity in breast cancer patients treated with single shot partial breast irradiation. ${ }^{35}$ Terrazzino et al, in a retrospective study, evaluated the association between the risk of subcutaneous fibrosis in irradiated breast cancer patients and eight polymorphic variants in six candidate genes related to DNA repair mechanisms, oxidative stress response, and fibroblast proliferation and differentiation. The results suggested that polymorphisms in GSTP1, GSTA1, and TGFB1 might contribute to the occurrence of grade 2-3 skin fibrosis (graded with SOMA-LENT system). ${ }^{36}$

Although data regarding the association of these SNPs and post-radiation late normal tissue injury have been controversial, we could certainly conclude that the combination of heterozygous mutations in XRCC1 G28152A (T/C) and XRCC3 C18067T (A/G) and homozygous mutation in GSTP1 A313G $(\mathrm{G} / \mathrm{G})$ may have played important roles in the development of severe late tissue toxicity in our patient.

\section{Conclusion}

When a severe subcutaneous or cutaneous post-radiation fibrosis develops in the absence of pathological or technical explanations, tests for SNPs involving DNA repair genes, such as GSTP1 (A313G), XRCC1 (G28152A), XRCC3 (A4541G and C18067T), and RAD51 (G135C), should be considered. Knowledge of the molecular basis of radiosensitivity could be useful in identifying extremely radiosensitive individuals who may not tolerate RT as a treatment option or to exclude these predisposed patients from dose escalation or hypofractionation.

\section{Disclosure}

The authors report no conflicts of interest in this work.

\section{References}

1. Early Breast Cancer Trialists' Collaborative Group (EBCTCG), Darby S, McGale P, Correa C, et al. Effect of radiotherapy after breast-conserving surgery on 10-year recurrence and 15-year breast cancer death: metaanalysis of individual patient data for 10,801 women in 17 randomised trials. Lancet. 2011;378(9804):1707-1716.

2. Bentzen SM. Preventing or reducing late side effects of radiation therapy: radiobiology meets molecular pathology. Nat Rev Cancer. 2006; 6(9):702-713.

3. Skoczylas JZ, Bentzen SM, Overgaard M, Overgaard J. Time course of radiobiological lung density changes after postmastectomy radiotherapy. Acta Oncol. 2000;39(2):181-187.

4. Bentzen SM, Skoczylas JZ, Overgaard M, Overgaard J. Radiotherapyrelated lung fibrosis enhanced by tamoxifen. J Natl Cancer Inst. 1996;88(13):918-922.

5. Altinok AY, Yildirim S, Altug T, et al. Aromatase inhibitors decrease radiation-induced lung fibrosis: results of an experimental study. Breast. 2016;28:174-177.

6. Denekamp J, Bartelink H, Rubin P. Correction for the use of SOMA LENT tables. Int J Radiat Oncol Biol Phys. 1996;35(2):417.

7. Litière S, Werutsky G, Fentiman IS, et al. Breast conserving therapy versus mastectomy for stage I-II breast cancer: 20 year follow-up of the EORTC 10801 phase 3 randomised trial. Lancet Oncol. 2012;13(4): 412-419.

8. Stone HB, Coleman CN, Anscher MS, McBride WH. Effects of radiation on normal tissue: consequences and mechanisms. Lancet Oncol. 2003;4(9):529-536. 
9. Rübe CE, Dong X, Kühne M, et al. DNA double- strand break rejoining in complex normal tissues. Int J Radiat Oncol Biol Phys. 2008;72(4):1180-1187.

10. Baumann M, Bentzen SM. Clinical manifestations of normal tissue damage. In: Steel GG, editor. Basic Clinical Radiobiology. London: Edward Arnold; 2002:116.

11. Bentzen SM, Overgaard M, Thames HD, Christensen JJ, Overgaard J. Early and late normal tissue injury after postmastectomy radiotherapy alone or combined with chemotherapy. Int J Radiat Biol. 1989;56(5):711-715.

12. Leadon SA. Repair of DNA damage produced by ionizing radiation: a minireview. Semin Radiat Oncol. 1996;6(4):295-305.

13. Haimovitz-Friedman A. Radiation-induced signal transduction and stress response. Radiat Res. 1998;150(5 Suppl):S102-S108.

14. Murray D, Begg AD. DNA repair genes and radiosensitivity. In: Panasci LC, Alaoui-Jamali MA, editors. DNA Repair in Cancer Therapy. Totowa, NJ: Humana Press; 2004:211-256.

15. Kerns SL, West CM, Andreassen CN, et al. Radiogenomics: the search for genetic predictors of radiotherapy response. Future Oncol. 2014;10(15): 2391-2406.

16. Vidal AE, Boiteux S, Hickson ID, Radicella JP. XRCC1 coordinates the initial and late stages of DNA abasic site repair through protein-protein interactions. EMBO J. 2001;20(22):6530-6539.

17. HuangY,Li L, Yu L. XRCC1,Arg399Gln, Arg194Trp and Arg280His polymorphisms in breast cancer risk: a meta-analysis. Mutagenesis. 2009;24(4): 331-339.

18. Li H, Ha TC, Tai BC. XRCC1 gene polymorphisms and breast cancer risk in different populations: a meta-analysis. Breast. 2009;18(3):183-191.

19. Moullan N, Cox DG, Angèle S, Romestaing P, Gèrard JP, Hall J. Polymorphisms in the DNA repair gene XRCC1, breast cancer risk, and response to radiotherapy. Cancer Epidemiol Biomarkers Prev. 2003;12(11 Pt 1):1168-1174.

20. Xie XX, Ouyang SY, Jin HK, Wang H, Zhou JM, Hu BQ. Predictive value of Xrcc1 gene polymorphisms for side effects in patients undergoing whole breast radiotherapy: a meta-analysis. Asian Pac J Cancer Prev. 2012;13(12):6121-6128.

21. Chang-Claude J, Popanda O, Tan XL, et al. Association between polymorphisms in the DNA repair genes, XRCC1, APE1, and XPD and acute side effects of radiotherapy in breast cancer patients. Clin Cancer Res. 2005;11(13):4802-4809.

22. Chang-Claude J, Ambrosone CB, Lilla C, et al. Genetic polymorphisms in DNA repair and damage response genes and late normal tissue complications of radiotherapy for breast cancer. $\mathrm{Br} J$ Cancer. 2009;100(10):1680-1686.

23. Andreassen CN, Alsner J, Overgaard M, Overgaard J. Prediction of normal tissue radiosensitivity from polymorphisms in candidate genes. Radiother Oncol. 2003;69(2):127-135.
24. Andreassen CN, Dikomey E, Parliament M, West CM. Will SNPs be useful predictors of normal tissue radiosensitivity in the future? Radiother Oncol. 2012;105(3):283-288.

25. Seibold $\mathrm{P}$, Behrens $\mathrm{S}, \mathrm{Schmezer} \mathrm{P}$, et al. XRCC1 polymorphism associated with late toxicity after radiation therapy in breast cancer patients. Int J Radiat Oncol Biol Phys. 2015;92(5):1084-1092.

26. Barnett GC, Coles CE, Elliott RM, et al. Independent validation of genes and polymorphisms reported to be associated with radiation toxicity: a prospective analysis study. Lancet Oncol. 2012;13(1): 65-77.

27. Azria D, Ozsahin M, Kramar A, et al. Single nucleotide polymorphisms, apoptosis, and the development of severe late adverse effects after radiotherapy. Clin Cancer Res. 2008;14(19):6284-6288.

28. Giotopoulos G, Symonds RP, Foweraker K, et al. The late radiotherapy normal tissue injury phenotypes of telangiectasia, fibrosis and atrophy in breast cancer patients have distinct genotype-dependent causes. $\mathrm{Br}$ J Cancer. 2007;96(6):1001-1007.

29. Jackson SP. Sensing and repairing DNA double-strand breaks. Carcinogenesis. 2002;23(5):687-696.

30. Dikomey E, Dahm-Daphi J, Brammer I, Martensen R, Kaina B. Correlation between cellular radiosensitivity and non-repaired doublestrand breaks studied in nine mammalian cell lines. Int J Radiat Biol. 1998;73(3):269-278.

31. Shen MR, Jones IM, Mohrenweiser H. Nonconservative amino acid substitution variants exist at polymorphic frequency in DNA repair genes in healthy humans. Cancer Res. 1998;58(4):604-608.

32. Mao CF, Qian WY, Wu JZ, Sun DW, Tang JH. Association between the XRCC3 Thr241Met polymorphism and breast cancer risk: an updated meta-analysis of 36 case-control studies. Asian Pac J Cancer Prev. 2014;15(16):6613-6618.

33. Song YZ, Han FJ, Liu M, Xia CC, Shi WY, Dong LH. Association between single nucleotide polymorphisms in XRCC3 and radiationinduced adverse effects on normal tissue: a meta-analysis. PLoS One. 2015;10(6):e0130388

34. Sundberg K, Johansson AS, Stenberg G, et al. Differences in the catalytic efficiencies of allelic variants of glutathione transferase P1-1 towards carcinogenic diol epoxides of polycyclic aromatic hydrocarbons. Carcinogenesis. 1998;19(3):433-436.

35. Falvo E, Strigari L, Citro G, et al. SNPs in DNA repair or oxidative stress genes and late subcutaneous fibrosis in patients following single shot partial breast irradiation. J Exp Clin Cancer Res. 2012;31(1):7.

36. Terrazzino S, La Mattina P, Gambaro G, et al. Common variants of GSTP1, GSTA1 and TGF $\beta 1$ are associated with the risk of radiationinduced fibrosis in breast cancer patients. Int J Radiat Oncol Biol Phys. 2012;83(2):504-511.
Breast Cancer - Targets and Therapy

\section{Publish your work in this journal}

Breast Cancer - Targets and Therapy is an international, peerreviewed open access journal focusing on breast cancer research, identification of therapeutic targets and the optimal use of preventative and integrated treatment interventions to achieve improved outcomes, enhanced survival and quality of life for the cancer patient.
Dovepress

The manuscript management system is completely online and includes a very quick and fair peer-review system, which is all easy to use. Visit http://www.dovepress.com/testimonials.php to read real quotes from published authors. 